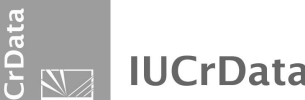

ISSN 2414-3146

Received 30 September 2017

Accepted 2 October 2017

Edited by J. Simpson, University of Otago, New Zealand

Keywords: crystal structure; quinoxaline; hydrazide; hydrogen bond.

CCDC reference: 1577756

Structural data: full structural data are available from iucrdata.iucr.org

\section{2-\{3-[2-(2-Chlorophenyl)ethyl]-2-oxo-1,2-dihydro- quinoxalin-1-yl\}acetohydrazide}

\author{
Youssef Ramli, ${ }^{a}$ Mohcine Missioui, ${ }^{\mathrm{b}, \mathrm{a} *}$ Mohammed El Fal, ${ }^{\mathrm{a}}$ Mohammed Ouhcine, \\ El Mokhtar Essassic and Joel T Mague ${ }^{\mathrm{d}}$
}

\begin{abstract}
aLaboratory of Medicinal Chemistry, Faculty of Medicine and Pharmacy, Mohammed V University, Rabat, Morocco, baboratory of Agrophysiology, Biotechnology, Environnement and Quality, Faculty of Science, Ibn Tofail University, Kenitra, Morocco, 'Laboratoire de Chimie Organique Heterocyclique URAC 21, Av. Ibn Battouta, BP 1014, Faculte des Sciences, Universite Mohammed V, Rabat, Morocco, and d Department of Chemistry, Tulane University, New Orleans, LA 70118, USA. *Correspondence e-mail: mohcinemissioui@yahoo.com
\end{abstract}

In the title compound, $\mathrm{C}_{18} \mathrm{H}_{17} \mathrm{ClN}_{4} \mathrm{O}_{2}$, the dihydroquinoxaline moiety deviates slightly from planarity. The benzene ring and its chloro and methylene substituents are disordered over two sets of sites, with an occupancy ratio of 0.675 (3):0.325 (3). In the crystal, corrugated sheets parallel to (100) are formed by $\mathrm{N}-\mathrm{H} \cdots \mathrm{O}, \mathrm{N}-\mathrm{H} \cdots \mathrm{Cl}$ and $\mathrm{N}-\mathrm{H} \cdots \mathrm{N}$ hydrogen bonds. The structure was refined as a two-component inversion twin.
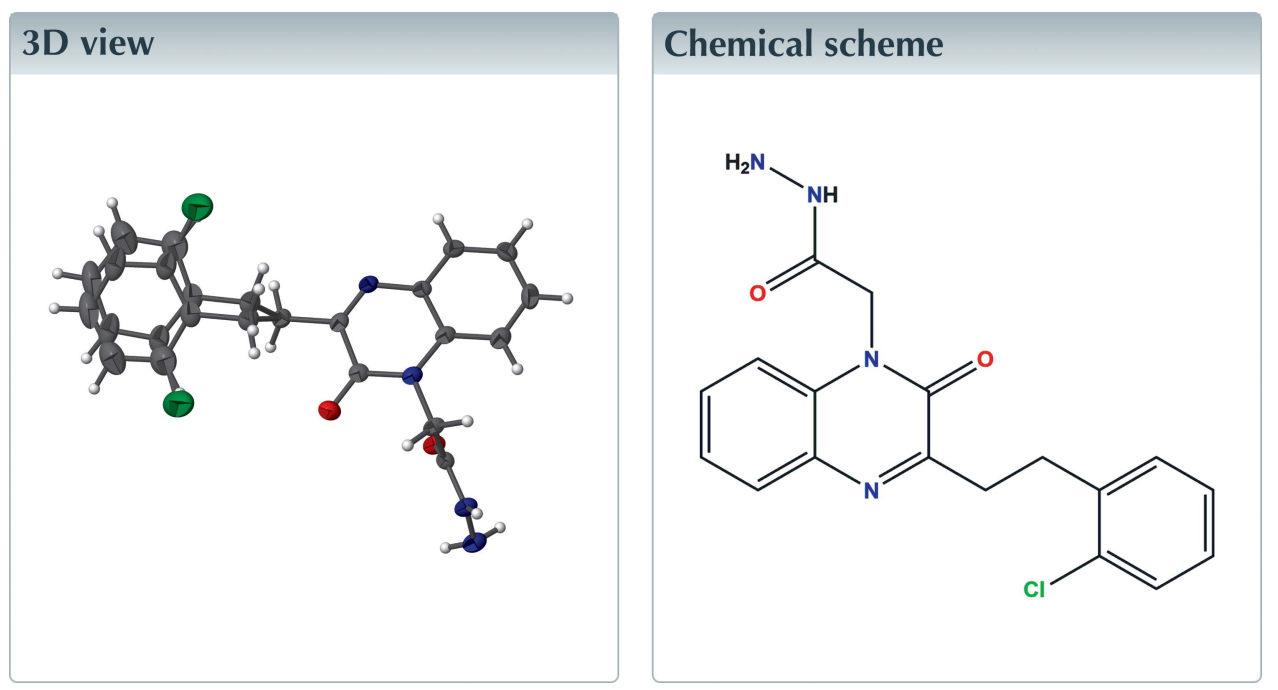

\section{Structure description}

Among the various classes of nitrogen heterocyclic compounds, quinoxaline derivatives display a broad spectrum of biological activities (Ramli et al., 2014). Some analogs have been synthesized and evaluated for their antimicrobial activity and many possess diverse biological activities including insecticidal, fungicidal, herbicidal, anthelmintic and antiviral (Ramli \& Essassi, 2015). As a continuation of our work on the development of Nsubstituted quinoxaline-2-one derivatives in order to evaluate their pharmacological activity, we have studied the condensation reaction of ethyl 2-[3-(2-chlorophenethyl)-2oxoquinoxalin-1(2H)-yl]acetate with hydrazine hydrate in ethanol to form the title compound (Fig. 1) in good yield (Ramli et al., 2011, 2013; Caleb et al., 2016).

The bicyclic core of the title compound is not quite planar, as indicated by the dihedral angle of $1.52(16)^{\circ}$ between the pyrazinone and the benzene rings. The pyrazinone ring is inclined to the major disorder component of the chlorophenyl ring by $24.80(19)^{\circ}$. In the crystal, the molecules form zigzag chains running along the $c$-axis direction through $\mathrm{N} 4-$ $\mathrm{H} 4 A \cdots \mathrm{N} 2$ hydrogen bonds together with weak $\mathrm{N} 4-\mathrm{H} 4 B \cdots \mathrm{Cl} 1$ interactions (Table 1 and 


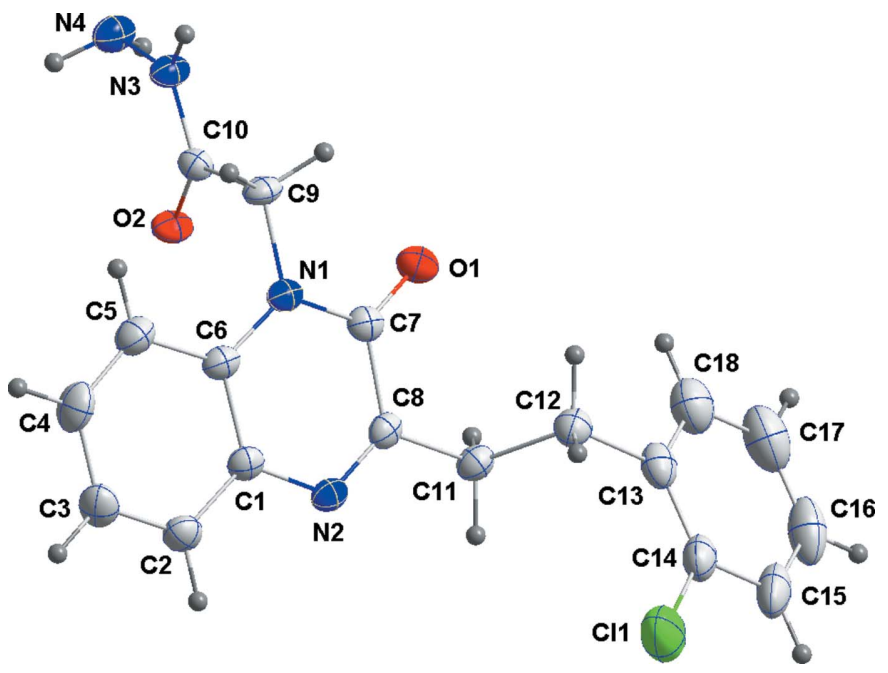

Figure 1

The title molecule with the labelling scheme and 50\% probability displacement ellipsoids. Only the major disorder component is shown for clarity.

Fig. 2). $\mathrm{N} 3-\mathrm{H} 3 A \cdots \mathrm{O} 2$ hydrogen bonds form chains along $b$ (Table 1 and Fig. 2) and combine with the sheets shown in Fig. 3 to form corrugated sheets parallel to (100).

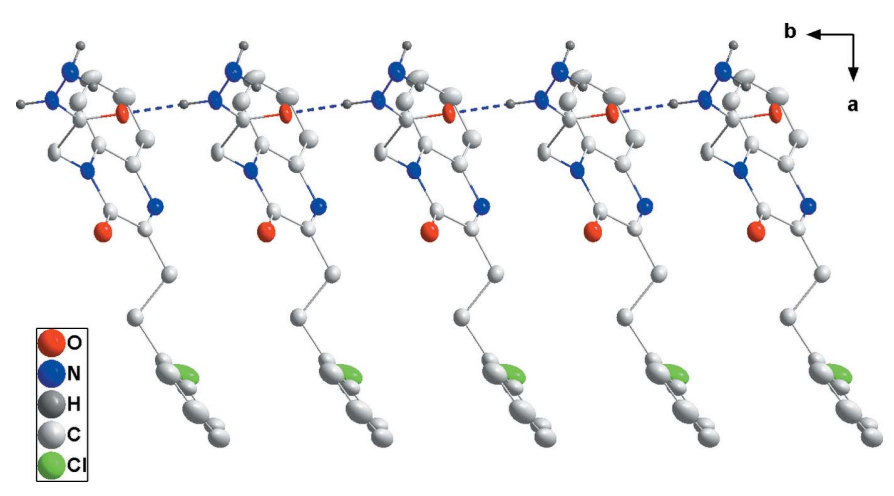

Figure 2

Chains of molecules formed along $b$ by $\mathrm{N}-\mathrm{H} \cdots \mathrm{O}$ hydrogen bonds.

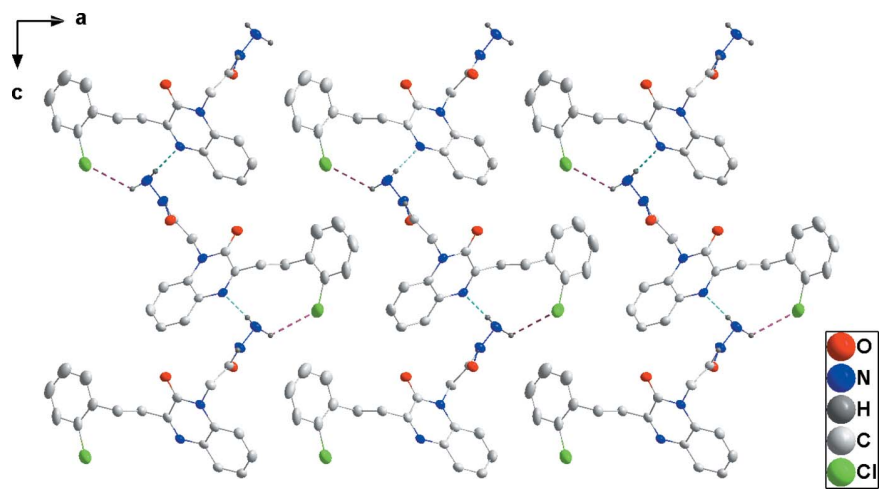

Figure 3

A view of the zigzag chains formed by $\mathrm{N}-\mathrm{H} \cdots \mathrm{N}$ (light-blue dashed lines) and $\mathrm{N}-\mathrm{H} \cdots \mathrm{Cl}$ (purple dashed lines) hydrogen bonds projected onto [010].
Table 1

Hydrogen-bond geometry $\left(\AA{ }^{\circ}\right)$.

\begin{tabular}{lllll}
\hline$D-\mathrm{H} \cdots A$ & $D-\mathrm{H}$ & $\mathrm{H} \cdots A$ & $D \cdots A$ & $D-\mathrm{H} \cdots A$ \\
\hline $\mathrm{N} 3-\mathrm{H} 3 A \cdots \mathrm{O} 2^{\mathrm{i}}$ & 0.91 & 1.96 & $2.842(4)$ & 163 \\
$\mathrm{~N} 4-\mathrm{H} 4 A \cdots \mathrm{N} 2^{i \mathrm{i}}$ & 0.91 & 2.35 & $3.256(4)$ & 175 \\
$\mathrm{~N}^{-\mathrm{H}} 4 B \cdots \mathrm{Cl}^{\mathrm{ii}}$ & 0.91 & 2.92 & $3.539(3)$ & 126 \\
\hline
\end{tabular}

Symmetry codes: (i) $x, y+1, z$; (ii) $-x+1,-y+1, z-\frac{1}{2}$.

Table 2

Experimental details.

\begin{tabular}{|c|c|}
\hline \multicolumn{2}{|l|}{ Crystal data } \\
\hline Chemical formula & $\mathrm{C}_{18} \mathrm{H}_{17} \mathrm{ClN}_{4} \mathrm{O}_{2}$ \\
\hline$M_{\mathrm{r}}$ & 356.80 \\
\hline Crystal system, space group & Orthorhombic, Pna2 1 \\
\hline Temperature (K) & 100 \\
\hline$a, b, c(\AA)$ & $24.258(3), 4.6484(5), 14.7708(16)$ \\
\hline$V\left(\AA^{3}\right)$ & $1665.6(3)$ \\
\hline$Z$ & 4 \\
\hline Radiation type & Mo $K \alpha$ \\
\hline$\mu\left(\mathrm{mm}^{-1}\right)$ & 0.25 \\
\hline Crystal size $(\mathrm{mm})$ & $0.25 \times 0.16 \times 0.05$ \\
\hline
\end{tabular}

Data collection

Diffractometer

Absorption correction

Bruker SMART APEX CCD

Multi-scan (SADABS; Bruker, 2016)

$T_{\min }, T_{\max }$

$0.94,0.99$

No. of measured, independent and observed $[I>2 \sigma(I)]$ reflections

$R_{\text {int }}$

$7372,4036,3379$

$(\sin \theta / \lambda)_{\max }\left(\AA^{-1}\right)$

0.027

0.668

Refinement

$R\left[F^{2}>2 \sigma\left(F^{2}\right)\right], w R\left(F^{2}\right), S \quad 0.052,0.145,1.07$

No. of reflections

4036

No. of parameters

231

No. of restraints

84

$\mathrm{H}$-atom treatment

$\mathrm{H}$ atoms treated by a mixture of independent and constrained refinement

$\Delta \rho_{\max }, \Delta \rho_{\min }\left(\mathrm{e} \AA^{-3}\right)$

Absolute structure

$0.77,-0.30$

Refined as an inversion twin

Absolute structure parameter $0.49(16)$

Computer programs: APEX3 and SAINT (Bruker, 2016), SHELXT (Sheldrick, 2015a), SHELXL2014 (Sheldrick, 2015b), DIAMOND (Brandenburg \& Putz, 2012) and SHELXTL (Sheldrick, 2008).

\section{Synthesis and crystallization}

To a solution of ethyl 2-[3-(2-chlorophenethyl)-2-oxoquinoxalin-1 $(2 H)$-yl]acetate $(2.70 \mathrm{mmol}, 1 \mathrm{~g})$ in $20 \mathrm{ml}$ of ethanol, hydrazine hydrate $(4.58 \mathrm{mmol}, 229.49 \mathrm{mg})$ was added. The mixture was stirred at room temperature for $24 \mathrm{~h}$. The solid material was removed by filtration and the solvent evaporated under vacuum. The solid product was purified by recrystallization from ethanol solution to afford colorless block-like crystals of the title compound (yield 63\%).

\section{Refinement}

Crystal and refinement details are presented in Table 2. The 2-chlorobenzyl group is disordered over several closely spaced positions. After several attempts, the only feasible model was a two-site one, treating the rings as rigid hexagons. The structure was refined as a two-component inversion twin. 


\section{Acknowledgements}

JTM thanks Tulane University for support of the Tulane Crystallography Laboratory.

\section{References}

Brandenburg, K. \& Putz, H. (2012). DIAMOND. Crystal Impact GbR, Bonn, Germany.

Bruker (2016). APEX3, SADABS and SAINT. Bruker AXS Inc., Madison, Wisconsin, USA.
Caleb, A. A., Ramli, Y., Benabdelkame, H., Bouhfid, R., Es-Safi, N., Kandri Rodi, Y., Essassi, E. M. \& Banoub, J. (2016). J. Mar. Chim. Heterocycl, 15, 109-123.

Ramli, Y. \& Essassi, E. M. (2015). Adv. Chem. Res. 27, 109-160.

Ramli, Y., Karrouchi, K., Essassi, E. M. \& El Ammari, L. (2013). Acta Cryst. E69, o1320-01321.

Ramli, Y., Moussaif, A., Karrouchi, K. \& Essassi, E. M. (2014). J. Chem. Article ID 563406, 1-21.

Ramli, Y., Moussaif, A., Zouihri, H., Bourichi, H. \& Essassi, E. M. (2011). Acta Cryst. E67, o1374.

Sheldrick, G. M. (2008). Acta Cryst. A64, 112-122.

Sheldrick, G. M. (2015a). Acta Cryst. A71, 3-8.

Sheldrick, G. M. (2015b). Acta Cryst. C71, 3-8. 


\section{full crystallographic data}

IUCrData (2017). 2, x171424 [https://doi.org/10.1107/S2414314617014249]

\section{2-\{3-[2-(2-Chlorophenyl)ethyl]-2-oxo-1,2-dihydroquinoxalin-1-}

\section{yl\}acetohydrazide}

Youssef Ramli, Mohcine Missioui, Mohammed El Fal, Mohammed Ouhcine, El Mokhtar Essassi and Joel T Mague

2-\{3-[2-(2-Chlorophenyl)ethyl]-2-oxo-1,2-dihydroquinoxalin-1-yl\}acetohydrazide

Crystal data

$\mathrm{C}_{18} \mathrm{H}_{17} \mathrm{ClN}_{4} \mathrm{O}_{2}$

$M_{r}=356.80$

Orthorhombic, Pna2 $_{1}$

$a=24.258(3) \AA$

$b=4.6484(5) \AA$

$c=14.7708(16) \AA$

$V=1665.6(3) \AA^{3}$

$Z=4$

$F(000)=744$

Data collection

Bruker SMART APEX CCD diffractometer

Radiation source: fine-focus sealed tube

Graphite monochromator

Detector resolution: 8.3333 pixels $\mathrm{mm}^{-1}$

$\omega$ scans

Absorption correction: multi-scan

(SADABS; Bruker, 2016)

$T_{\min }=0.94, T_{\max }=0.99$

Refinement

Refinement on $F^{2}$

Least-squares matrix: full

$R\left[F^{2}>2 \sigma\left(F^{2}\right)\right]=0.052$

$w R\left(F^{2}\right)=0.145$

$S=1.07$

4036 reflections

231 parameters

84 restraints

Primary atom site location: structure-invariant direct methods
$D_{\mathrm{x}}=1.423 \mathrm{Mg} \mathrm{m}^{-3}$

Mo $K \alpha$ radiation, $\lambda=0.71073 \AA$

Cell parameters from 6865 reflections

$\theta=2.2-27.5^{\circ}$

$\mu=0.25 \mathrm{~mm}^{-1}$

$T=100 \mathrm{~K}$

Block, colorless

$0.25 \times 0.16 \times 0.05 \mathrm{~mm}$

7372 measured reflections

4036 independent reflections

3379 reflections with $I>2 \sigma(I)$

$R_{\text {int }}=0.027$

$\theta_{\max }=28.3^{\circ}, \theta_{\min }=1.7^{\circ}$

$h=-32 \rightarrow 32$

$k=-6 \rightarrow 6$

$l=-19 \rightarrow 19$

Secondary atom site location: difference Fourier map

Hydrogen site location: mixed

$\mathrm{H}$ atoms treated by a mixture of independent and constrained refinement

$w=1 /\left[\sigma^{2}\left(F_{\mathrm{o}}^{2}\right)+(0.0818 P)^{2}+0.3986 P\right]$ where $P=\left(F_{\mathrm{o}}{ }^{2}+2 F_{\mathrm{c}}{ }^{2}\right) / 3$

$(\Delta / \sigma)_{\max }<0.001$

$\Delta \rho_{\max }=0.77 \mathrm{e}^{-3}$

$\Delta \rho_{\min }=-0.30$ e $\AA^{-3}$

Absolute structure: Refined as an inversion twin

Absolute structure parameter: 0.49 (16) 


\section{Special details}

Experimental. The diffraction data were collected in three sets of 363 frames $\left(0.5^{\circ}\right.$ width in $\left.\omega\right)$ at $\varphi=0,120$ and $240^{\circ}$. A scan time of $180 \mathrm{sec} /$ frame was used.

Geometry. All esds (except the esd in the dihedral angle between two 1.s. planes) are estimated using the full covariance matrix. The cell esds are taken into account individually in the estimation of esds in distances, angles and torsion angles; correlations between esds in cell parameters are only used when they are defined by crystal symmetry. An approximate (isotropic) treatment of cell esds is used for estimating esds involving l.s. planes.

Refinement. Refinement of $\mathrm{F}^{2}$ against ALL reflections. The weighted R-factor $\mathrm{wR}$ and goodness of fit $\mathrm{S}$ are based on $\mathrm{F}^{2}$, conventional $\mathrm{R}$-factors $\mathrm{R}$ are based on $\mathrm{F}$, with $\mathrm{F}$ set to zero for negative $\mathrm{F}^{2}$. The threshold expression of $\mathrm{F}^{2}>2 \operatorname{sigma}\left(\mathrm{F}^{2}\right)$ is used only for calculating R-factors(gt) etc. and is not relevant to the choice of reflections for refinement. R-factors based on $\mathrm{F}^{2}$ are statistically about twice as large as those based on F, and R- factors based on ALL data will be even larger. The 2-chlorobenzyl group is disordered over several closely spaced positions. After several attempts, the only feasible model was a 2 -site one treating the rings as rigid hexagons. H-atoms attached to carbon were placed in idealized positions while those attached to nitrogen were placed in locations derived from a difference map and their coordinates adjusted to give $\mathrm{N}-\mathrm{H}=0.91 \% \mathrm{~A}$. All were included as riding contributions. Refined as a 2-component inversion twin.

Fractional atomic coordinates and isotropic or equivalent isotropic displacement parameters $\left(\AA^{2}\right)$

\begin{tabular}{|c|c|c|c|c|c|}
\hline & $x$ & $y$ & $z$ & $U_{\text {iso }} * / U_{\text {eq }}$ & Occ. $(<1)$ \\
\hline $\mathrm{O} 1$ & $0.57433(10)$ & $0.7964(6)$ & $0.42015(17)$ & $0.0305(6)$ & \\
\hline $\mathrm{O} 2$ & $0.43551(10)$ & $0.6782(5)$ & $0.38490(17)$ & $0.0267(5)$ & \\
\hline N1 & $0.50239(11)$ & $0.8913(6)$ & $0.51467(18)$ & $0.0231(6)$ & \\
\hline N2 & $0.54455(12)$ & $0.4766(6)$ & $0.63241(19)$ & $0.0242(6)$ & \\
\hline N3 & $0.41985(12)$ & $1.1092(6)$ & $0.3195(2)$ & $0.0263(6)$ & \\
\hline H3A & 0.4245 & 1.3014 & 0.3283 & $0.034(11)^{*}$ & \\
\hline N4 & $0.38670(13)$ & $0.9972(7)$ & $0.2490(2)$ & $0.0317(7)$ & \\
\hline $\mathrm{H} 4 \mathrm{~A}$ & 0.4043 & 0.8569 & 0.2173 & $0.044(13)^{*}$ & \\
\hline $\mathrm{H} 4 \mathrm{~B}$ & 0.3559 & 0.9345 & 0.2781 & $0.038(12)^{*}$ & \\
\hline $\mathrm{C} 1$ & $0.49487(13)$ & $0.6127(7)$ & $0.6508(2)$ & $0.0233(6)$ & \\
\hline $\mathrm{C} 2$ & $0.46541(15)$ & $0.5374(8)$ & $0.7293(2)$ & $0.0293(7)$ & \\
\hline $\mathrm{H} 2$ & 0.4799 & 0.3950 & 0.7688 & $0.035^{*}$ & \\
\hline $\mathrm{C} 3$ & $0.41607(16)$ & $0.6664(9)$ & $0.7498(2)$ & $0.0339(8)$ & \\
\hline H3 & 0.3969 & 0.6161 & 0.8036 & $0.041^{*}$ & \\
\hline $\mathrm{C} 4$ & $0.39449(15)$ & $0.8702(9)$ & $0.6914(3)$ & $0.0374(9)$ & \\
\hline $\mathrm{H} 4$ & 0.3602 & 0.9581 & 0.7056 & $0.045^{*}$ & \\
\hline $\mathrm{C} 5$ & $0.42163(15)$ & $0.9485(8)$ & $0.6132(3)$ & $0.0312(8)$ & \\
\hline H5 & 0.4059 & 1.0881 & 0.5740 & $0.037^{*}$ & \\
\hline C6 & $0.47239(14)$ & $0.8223(7)$ & $0.5917(2)$ & $0.0234(7)$ & \\
\hline $\mathrm{C} 7$ & $0.55016(14)$ & $0.7508(7)$ & $0.4917(2)$ & $0.0234(6)$ & \\
\hline $\mathrm{C} 8$ & $0.57052(13)$ & $0.5406(7)$ & $0.5594(2)$ & $0.0237(7)$ & \\
\hline C9 & $0.48067(14)$ & $1.0949(7)$ & $0.4481(2)$ & $0.0246(7)$ & \\
\hline H9A & 0.5116 & 1.1848 & 0.4147 & $0.030^{*}$ & \\
\hline H9B & 0.4600 & 1.2491 & 0.4795 & $0.030^{*}$ & \\
\hline $\mathrm{C} 10$ & $0.44243(13)$ & $0.9402(7)$ & $0.3812(2)$ & $0.0215(6)$ & \\
\hline $\mathrm{C} 11$ & $0.62385(13)$ & $0.3913(7)$ & $0.5368(2)$ & $0.0261(7)$ & \\
\hline H11A & 0.6205 & 0.3004 & 0.4764 & $0.031^{*}$ & \\
\hline H11B & 0.6305 & 0.2368 & 0.5816 & $0.031^{*}$ & \\
\hline $\mathrm{Cl} 1$ & $0.74190(7)$ & $0.3151(6)$ & 0.69305 (14) & $0.0692(6)$ & 0.675 \\
\hline $\mathrm{C} 12$ & $0.6744(3)$ & $0.6004(17)$ & $0.5363(5)$ & $0.0297(18)$ & $0.675(3)$ \\
\hline
\end{tabular}




$\begin{array}{llllll}\text { H12A } & 0.6689 & 0.7525 & 0.4903 & 0.036^{*} & 0.675(3) \\ \text { H12B } & 0.6783 & 0.6934 & 0.5963 & 0.036^{*} & 0.675(3) \\ \text { C13 } & 0.72605(16) & 0.4260(10) & 0.5144(3) & 0.0332(14) & 0.675(3) \\ \text { C14 } & 0.75662(17) & 0.2843(10) & 0.5803(3) & 0.0384(14) & 0.675(3) \\ \text { C15 } & 0.80138(15) & 0.1150(10) & 0.5555(4) & 0.0494(16) & 0.675(3) \\ \text { H15 } & 0.8223 & 0.0182 & 0.6005 & 0.059^{*} & 0.675(3) \\ \text { C16 } & 0.81556(16) & 0.0875(11) & 0.4647(4) & 0.054(2) & 0.675(3) \\ \text { H16 } & 0.8462 & -0.0281 & 0.4477 & 0.065^{*} & 0.675(3) \\ \text { C17 } & 0.7850(2) & 0.2293(13) & 0.3988(3) & 0.061(2) & 0.675(3) \\ \text { H17 } & 0.7947 & 0.2105 & 0.3367 & 0.073^{*} & 0.675(3) \\ \text { C18 } & 0.7402(2) & 0.3985(12) & 0.4236(3) & 0.0528(18) & 0.675(3) \\ \text { H18 } & 0.7193 & 0.4953 & 0.3786 & 0.063^{*} & 0.675(3) \\ \text { C11A } & 0.71901(15) & 0.5143(12) & 0.3615(3) & 0.0692(6) & 0.325(3) \\ \text { C12A } & 0.6753(6) & 0.566(4) & 0.5630(14) & 0.0297(18) & 0.325(3) \\ \text { H12C } & 0.6766 & 0.7474 & 0.5279 & 0.036^{*} & 0.325(3) \\ \text { H12D } & 0.6742 & 0.6146 & 0.6282 & 0.036^{*} & 0.325(3) \\ \text { C13A } & 0.7262(4) & 0.383(2) & 0.5423(8) & 0.0332(14) & 0.325(3) \\ \text { C14A } & 0.7474(4) & 0.345(2) & 0.4556(7) & 0.0384(14) & 0.325(3) \\ \text { C15A } & 0.7933(4) & 0.171(2) & 0.4423(6) & 0.0494(16) & 0.325(3) \\ \text { H15A } & 0.8077 & 0.1451 & 0.3830 & 0.059^{*} & 0.325(3) \\ \text { C16A } & 0.8180(4) & 0.035(2) & 0.5155(8) & 0.054(2) & 0.325(3) \\ \text { H16A } & 0.8494 & -0.0840 & 0.5064 & 0.065^{*} & 0.325(3) \\ \text { C17A } & 0.7969(4) & 0.073(3) & 0.6022(7) & 0.061(2) & 0.325(3) \\ \text { H17A } & 0.8138 & -0.0203 & 0.6523 & 0.073^{*} & 0.325(3) \\ \text { C18A } & 0.7510(5) & 0.247(3) & 0.6156(6) & 0.0528(18) & 0.325(3) \\ \text { H18A } & 0.7365 & 0.2725 & 0.6748 & 0.063^{*} & 0.325(3) \\ & & & & & \end{array}$

Atomic displacement parameters $\left(\AA^{2}\right)$

\begin{tabular}{lllllll}
\hline & $U^{11}$ & $U^{22}$ & $U^{33}$ & $U^{12}$ & $U^{13}$ & $U^{23}$ \\
\hline O1 & $0.0359(13)$ & $0.0295(13)$ & $0.0259(12)$ & $-0.0008(10)$ & $0.0010(10)$ & $0.0006(10)$ \\
O2 & $0.0348(12)$ & $0.0148(10)$ & $0.0304(12)$ & $-0.0028(9)$ & $-0.0054(10)$ & $0.0007(9)$ \\
N1 & $0.0264(13)$ & $0.0183(12)$ & $0.0245(13)$ & $-0.0020(11)$ & $-0.0054(11)$ & $-0.0004(11)$ \\
N2 & $0.0259(13)$ & $0.0234(14)$ & $0.0232(13)$ & $0.0003(11)$ & $-0.0059(11)$ & $-0.0006(11)$ \\
N3 & $0.0324(14)$ & $0.0174(13)$ & $0.0292(15)$ & $-0.0031(11)$ & $-0.0085(11)$ & $0.0012(11)$ \\
N4 & $0.0362(16)$ & $0.0261(15)$ & $0.0328(16)$ & $-0.0027(13)$ & $-0.0106(13)$ & $-0.0001(12)$ \\
C1 & $0.0261(15)$ & $0.0233(15)$ & $0.0204(14)$ & $-0.0009(12)$ & $-0.0041(12)$ & $-0.0023(12)$ \\
C2 & $0.0308(18)$ & $0.0328(18)$ & $0.0244(16)$ & $-0.0013(14)$ & $-0.0047(13)$ & $0.0016(15)$ \\
C3 & $0.0319(18)$ & $0.045(2)$ & $0.0244(17)$ & $-0.0014(16)$ & $0.0015(14)$ & $-0.0027(16)$ \\
C4 & $0.0275(17)$ & $0.043(2)$ & $0.042(2)$ & $0.0081(16)$ & $0.0027(15)$ & $-0.0094(18)$ \\
C5 & $0.0309(18)$ & $0.0278(19)$ & $0.0349(19)$ & $0.0032(14)$ & $-0.0048(14)$ & $0.0001(14)$ \\
C6 & $0.0278(16)$ & $0.0187(15)$ & $0.0237(15)$ & $-0.0005(12)$ & $-0.0061(12)$ & $-0.0018(12)$ \\
C7 & $0.0272(16)$ & $0.0187(15)$ & $0.0243(15)$ & $-0.0028(12)$ & $-0.0031(11)$ & $-0.0032(12)$ \\
C8 & $0.0227(15)$ & $0.0202(15)$ & $0.0281(16)$ & $-0.0022(12)$ & $-0.0061(12)$ & $-0.0025(13)$ \\
C9 & $0.0303(16)$ & $0.0157(15)$ & $0.0278(16)$ & $0.0013(13)$ & $-0.0063(12)$ & $0.0034(12)$ \\
C10 & $0.0248(14)$ & $0.0176(15)$ & $0.0221(14)$ & $-0.0005(11)$ & $0.0016(12)$ & $-0.0009(12)$ \\
C11 & $0.0271(15)$ & $0.0237(16)$ & $0.0276(16)$ & $0.0028(13)$ & $-0.0033(13)$ & $-0.0019(13)$ \\
C11 & $0.0429(8)$ & $0.1067(17)$ & $0.0581(9)$ & $-0.0183(9)$ & $-0.0076(7)$ & $0.0194(11)$
\end{tabular}




\begin{tabular}{lllllll} 
C12 & $0.0289(18)$ & $0.025(3)$ & $0.036(5)$ & $0.0000(18)$ & $-0.001(3)$ & $0.000(3)$ \\
C13 & $0.0282(18)$ & $0.026(2)$ & $0.045(4)$ & $-0.0052(17)$ & $0.005(2)$ & $-0.004(3)$ \\
C14 & $0.023(2)$ & $0.035(3)$ & $0.057(4)$ & $-0.004(2)$ & $0.001(2)$ & $0.003(3)$ \\
C15 & $0.023(2)$ & $0.040(3)$ & $0.085(4)$ & $-0.003(2)$ & $0.000(3)$ & $0.002(3)$ \\
C16 & $0.033(2)$ & $0.043(3)$ & $0.086(6)$ & $-0.004(2)$ & $0.017(4)$ & $-0.023(4)$ \\
C17 & $0.051(3)$ & $0.062(4)$ & $0.068(4)$ & $-0.015(3)$ & $0.019(3)$ & $-0.014(3)$ \\
C18 & $0.043(3)$ & $0.053(4)$ & $0.062(4)$ & $-0.011(3)$ & $0.005(3)$ & $-0.001(3)$ \\
C11A & $0.0429(8)$ & $0.1067(17)$ & $0.0581(9)$ & $-0.0183(9)$ & $-0.0076(7)$ & $0.0194(11)$ \\
C12A & $0.0289(18)$ & $0.025(3)$ & $0.036(5)$ & $0.0000(18)$ & $-0.001(3)$ & $0.000(3)$ \\
C13A & $0.0282(18)$ & $0.026(2)$ & $0.045(4)$ & $-0.0052(17)$ & $0.005(2)$ & $-0.004(3)$ \\
C14A & $0.023(2)$ & $0.035(3)$ & $0.057(4)$ & $-0.004(2)$ & $0.001(2)$ & $0.003(3)$ \\
C15A & $0.023(2)$ & $0.040(3)$ & $0.085(4)$ & $-0.003(2)$ & $0.000(3)$ & $0.002(3)$ \\
C16A & $0.033(2)$ & $0.043(3)$ & $0.086(6)$ & $-0.004(2)$ & $0.017(4)$ & $-0.023(4)$ \\
C17A & $0.051(3)$ & $0.062(4)$ & $0.068(4)$ & $-0.015(3)$ & $0.019(3)$ & $-0.014(3)$ \\
C18A & $0.043(3)$ & $0.053(4)$ & $0.062(4)$ & $-0.011(3)$ & $0.005(3)$ & $-0.001(3)$ \\
& & & & & & \\
\hline
\end{tabular}

Geometric parameters $\left(A,{ }^{\circ}\right)$

\begin{tabular}{llll}
\hline $\mathrm{O} 1-\mathrm{C} 7$ & $1.227(4)$ & $\mathrm{C} 11-\mathrm{H} 11 \mathrm{~B}$ & 0.9900 \\
$\mathrm{O} 2-\mathrm{C} 10$ & $1.231(4)$ & $\mathrm{C} 1-\mathrm{C} 14$ & $1.709(4)$ \\
$\mathrm{N} 1-\mathrm{C} 7$ & $1.373(4)$ & $\mathrm{C} 12-\mathrm{C} 13$ & $1.528(8)$ \\
$\mathrm{N} 1-\mathrm{C} 6$ & $1.389(4)$ & $\mathrm{C} 12-\mathrm{H} 12 \mathrm{~A}$ & 0.9900 \\
$\mathrm{~N} 1-\mathrm{C} 9$ & $1.463(4)$ & $\mathrm{C} 12-\mathrm{H} 12 \mathrm{~B}$ & 0.9900 \\
$\mathrm{~N} 2-\mathrm{C} 8$ & $1.284(5)$ & $\mathrm{C} 13-\mathrm{C} 14$ & 1.3900 \\
$\mathrm{~N} 2-\mathrm{C} 1$ & $1.388(4)$ & $\mathrm{C} 13-\mathrm{C} 18$ & 1.3900 \\
$\mathrm{~N} 3-\mathrm{C} 10$ & $1.323(4)$ & $\mathrm{C} 14-\mathrm{C} 15$ & 1.3900 \\
$\mathrm{~N} 3-\mathrm{N} 4$ & $1.414(4)$ & $\mathrm{C} 15-\mathrm{C} 16$ & 1.3900 \\
$\mathrm{~N} 3-\mathrm{H} 3 \mathrm{~A}$ & 0.9099 & $\mathrm{C} 15-\mathrm{H} 15$ & 0.9500 \\
$\mathrm{~N} 4-\mathrm{H} 4 \mathrm{~A}$ & 0.9100 & $\mathrm{C} 16-\mathrm{C} 17$ & 1.3900 \\
$\mathrm{~N} 4-\mathrm{H} 4 \mathrm{~B}$ & 0.9100 & $\mathrm{C} 16-\mathrm{H} 16$ & 0.9500 \\
$\mathrm{C} 1-\mathrm{C} 2$ & $1.407(5)$ & $\mathrm{C} 17-\mathrm{C} 18$ & 1.3900 \\
$\mathrm{C} 1-\mathrm{C} 6$ & $1.416(4)$ & $\mathrm{C} 17-\mathrm{H} 17$ & 0.9500 \\
$\mathrm{C} 2-\mathrm{C} 3$ & $1.372(5)$ & $\mathrm{C} 18-\mathrm{H} 18$ & 0.9500 \\
$\mathrm{C} 2-\mathrm{H} 2$ & 0.9500 & $\mathrm{C} 11 \mathrm{~A}-\mathrm{C} 14 \mathrm{~A}$ & $1.740(9)$ \\
$\mathrm{C} 3-\mathrm{C} 4$ & $1.384(6)$ & $\mathrm{C} 12 \mathrm{~A}-\mathrm{C} 13 \mathrm{~A}$ & $1.533(18)$ \\
$\mathrm{C} 3-\mathrm{H} 3$ & 0.9500 & $\mathrm{C} 12 \mathrm{~A}-\mathrm{H} 12 \mathrm{C}$ & 0.9900 \\
$\mathrm{C} 4-\mathrm{C} 5$ & $1.378(6)$ & $\mathrm{C} 12 \mathrm{~A}-\mathrm{H} 12 \mathrm{D}$ & 0.9900 \\
$\mathrm{C} 4-\mathrm{H} 4$ & 0.9500 & $\mathrm{C} 13 \mathrm{~A}-\mathrm{C} 14 \mathrm{~A}$ & 1.3900 \\
$\mathrm{C} 5-\mathrm{C} 6$ & $1.401(5)$ & $\mathrm{C} 13 \mathrm{~A}-\mathrm{C} 18 \mathrm{~A}$ & 1.3900 \\
$\mathrm{C} 5-\mathrm{H} 5$ & 0.9500 & $\mathrm{C} 14 \mathrm{~A}-\mathrm{C} 15 \mathrm{~A}$ & 1.3900 \\
$\mathrm{C} 7-\mathrm{C} 8$ & $1.483(5)$ & $\mathrm{C} 15 \mathrm{~A}-\mathrm{C} 16 \mathrm{~A}$ & 1.3900 \\
$\mathrm{C} 8-\mathrm{C} 11$ & $1.506(4)$ & $\mathrm{C} 15 \mathrm{~A}-\mathrm{H} 15 \mathrm{~A}$ & 0.9500 \\
$\mathrm{C} 9-\mathrm{C} 10$ & $1.533(4)$ & $\mathrm{C} 16 \mathrm{~A}-\mathrm{C} 17 \mathrm{~A}$ & 1.3900 \\
$\mathrm{C} 9-\mathrm{H} 9 \mathrm{~A}$ & 0.9900 & $\mathrm{C} 16 \mathrm{~A}-\mathrm{H} 16 \mathrm{~A}$ & 0.9500 \\
$\mathrm{C} 9-\mathrm{H} 9 \mathrm{~B}$ & 0.9900 & $\mathrm{C} 17 \mathrm{~A}-\mathrm{C} 18 \mathrm{~A}$ & 1.3900 \\
$\mathrm{C} 11-\mathrm{C} 12 \mathrm{~A}$ & $1.538(16)$ & $\mathrm{C} 17 \mathrm{~A}-\mathrm{H} 17 \mathrm{~A}$ & 0.9500 \\
$\mathrm{C} 11-\mathrm{C} 12$ & $1.564(8)$ & $\mathrm{C} 18 \mathrm{~A}-\mathrm{H} 18 \mathrm{~A}$ & \\
$\mathrm{C} 11-\mathrm{H} 11 \mathrm{~A}$ & 0.9900 & &
\end{tabular}




\begin{tabular}{|c|c|c|c|}
\hline $\mathrm{C} 7-\mathrm{N} 1-\mathrm{C} 6$ & $122.4(3)$ & $\mathrm{H} 11 \mathrm{~A}-\mathrm{C} 11-\mathrm{H} 11 \mathrm{~B}$ & 107.8 \\
\hline $\mathrm{C} 7-\mathrm{N} 1-\mathrm{C} 9$ & $116.4(3)$ & $\mathrm{C} 13-\mathrm{C} 12-\mathrm{C} 11$ & $108.3(5)$ \\
\hline $\mathrm{C} 6-\mathrm{N} 1-\mathrm{C} 9$ & $120.8(3)$ & $\mathrm{C} 13-\mathrm{C} 12-\mathrm{H} 12 \mathrm{~A}$ & 110.0 \\
\hline $\mathrm{C} 8-\mathrm{N} 2-\mathrm{C} 1$ & $119.0(3)$ & $\mathrm{C} 11-\mathrm{C} 12-\mathrm{H} 12 \mathrm{~A}$ & 110.0 \\
\hline $\mathrm{C} 10-\mathrm{N} 3-\mathrm{N} 4$ & $121.6(3)$ & $\mathrm{C} 13-\mathrm{C} 12-\mathrm{H} 12 \mathrm{~B}$ & 110.0 \\
\hline $\mathrm{C} 10-\mathrm{N} 3-\mathrm{H} 3 \mathrm{~A}$ & 115.7 & $\mathrm{C} 11-\mathrm{C} 12-\mathrm{H} 12 \mathrm{~B}$ & 110.0 \\
\hline $\mathrm{N} 4-\mathrm{N} 3-\mathrm{H} 3 \mathrm{~A}$ & 122.5 & $\mathrm{H} 12 \mathrm{~A}-\mathrm{C} 12-\mathrm{H} 12 \mathrm{~B}$ & 108.4 \\
\hline $\mathrm{N} 3-\mathrm{N} 4-\mathrm{H} 4 \mathrm{~A}$ & 112.1 & $\mathrm{C} 14-\mathrm{C} 13-\mathrm{C} 18$ & 120.0 \\
\hline $\mathrm{N} 3-\mathrm{N} 4-\mathrm{H} 4 \mathrm{~B}$ & 103.7 & $\mathrm{C} 14-\mathrm{C} 13-\mathrm{C} 12$ & $122.7(4)$ \\
\hline $\mathrm{H} 4 \mathrm{~A}-\mathrm{N} 4-\mathrm{H} 4 \mathrm{~B}$ & 113.5 & $\mathrm{C} 18-\mathrm{C} 13-\mathrm{C} 12$ & $117.2(4)$ \\
\hline $\mathrm{N} 2-\mathrm{C} 1-\mathrm{C} 2$ & $119.2(3)$ & $\mathrm{C} 15-\mathrm{C} 14-\mathrm{C} 13$ & 120.0 \\
\hline $\mathrm{N} 2-\mathrm{C} 1-\mathrm{C} 6$ & $121.8(3)$ & $\mathrm{C} 15-\mathrm{C} 14-\mathrm{Cl} 1$ & $117.9(3)$ \\
\hline $\mathrm{C} 2-\mathrm{C} 1-\mathrm{C} 6$ & $118.9(3)$ & $\mathrm{C} 13-\mathrm{C} 14-\mathrm{Cl1}$ & $122.1(3)$ \\
\hline $\mathrm{C} 3-\mathrm{C} 2-\mathrm{C} 1$ & $121.0(3)$ & $\mathrm{C} 14-\mathrm{C} 15-\mathrm{C} 16$ & 120.0 \\
\hline $\mathrm{C} 3-\mathrm{C} 2-\mathrm{H} 2$ & 119.5 & $\mathrm{C} 14-\mathrm{C} 15-\mathrm{H} 15$ & 120.0 \\
\hline $\mathrm{C} 1-\mathrm{C} 2-\mathrm{H} 2$ & 119.5 & $\mathrm{C} 16-\mathrm{C} 15-\mathrm{H} 15$ & 120.0 \\
\hline $\mathrm{C} 2-\mathrm{C} 3-\mathrm{C} 4$ & $119.5(3)$ & $\mathrm{C} 17-\mathrm{C} 16-\mathrm{C} 15$ & 120.0 \\
\hline $\mathrm{C} 2-\mathrm{C} 3-\mathrm{H} 3$ & 120.3 & $\mathrm{C} 17-\mathrm{C} 16-\mathrm{H} 16$ & 120.0 \\
\hline $\mathrm{C} 4-\mathrm{C} 3-\mathrm{H} 3$ & 120.3 & $\mathrm{C} 15-\mathrm{C} 16-\mathrm{H} 16$ & 120.0 \\
\hline $\mathrm{C} 5-\mathrm{C} 4-\mathrm{C} 3$ & $121.5(3)$ & $\mathrm{C} 16-\mathrm{C} 17-\mathrm{C} 18$ & 120.0 \\
\hline $\mathrm{C} 5-\mathrm{C} 4-\mathrm{H} 4$ & 119.3 & $\mathrm{C} 16-\mathrm{C} 17-\mathrm{H} 17$ & 120.0 \\
\hline $\mathrm{C} 3-\mathrm{C} 4-\mathrm{H} 4$ & 119.3 & $\mathrm{C} 18-\mathrm{C} 17-\mathrm{H} 17$ & 120.0 \\
\hline $\mathrm{C} 4-\mathrm{C} 5-\mathrm{C} 6$ & $120.0(3)$ & $\mathrm{C} 17-\mathrm{C} 18-\mathrm{C} 13$ & 120.0 \\
\hline $\mathrm{C} 4-\mathrm{C} 5-\mathrm{H} 5$ & 120.0 & $\mathrm{C} 17-\mathrm{C} 18-\mathrm{H} 18$ & 120.0 \\
\hline $\mathrm{C} 6-\mathrm{C} 5-\mathrm{H} 5$ & 120.0 & $\mathrm{C} 13-\mathrm{C} 18-\mathrm{H} 18$ & 120.0 \\
\hline $\mathrm{N} 1-\mathrm{C} 6-\mathrm{C} 5$ & $123.4(3)$ & $\mathrm{C} 13 \mathrm{~A}-\mathrm{C} 12 \mathrm{~A}-\mathrm{C} 11$ & $108.1(11)$ \\
\hline $\mathrm{N} 1-\mathrm{C} 6-\mathrm{C} 1$ & $117.5(3)$ & $\mathrm{C} 13 \mathrm{~A}-\mathrm{C} 12 \mathrm{~A}-\mathrm{H} 12 \mathrm{C}$ & 110.1 \\
\hline $\mathrm{C} 5-\mathrm{C} 6-\mathrm{C} 1$ & $119.1(3)$ & $\mathrm{C} 11-\mathrm{C} 12 \mathrm{~A}-\mathrm{H} 12 \mathrm{C}$ & 110.1 \\
\hline $\mathrm{O} 1-\mathrm{C} 7-\mathrm{N} 1$ & $122.3(3)$ & $\mathrm{C} 13 \mathrm{~A}-\mathrm{C} 12 \mathrm{~A}-\mathrm{H} 12 \mathrm{D}$ & 110.1 \\
\hline $\mathrm{O} 1-\mathrm{C} 7-\mathrm{C} 8$ & $122.4(3)$ & $\mathrm{C} 11-\mathrm{C} 12 \mathrm{~A}-\mathrm{H} 12 \mathrm{D}$ & 110.1 \\
\hline $\mathrm{N} 1-\mathrm{C} 7-\mathrm{C} 8$ & $115.3(3)$ & $\mathrm{H} 12 \mathrm{C}-\mathrm{C} 12 \mathrm{~A}-\mathrm{H} 12 \mathrm{D}$ & 108.4 \\
\hline $\mathrm{N} 2-\mathrm{C} 8-\mathrm{C} 7$ & $123.8(3)$ & $\mathrm{C} 14 \mathrm{~A}-\mathrm{C} 13 \mathrm{~A}-\mathrm{C} 18 \mathrm{~A}$ & 120.0 \\
\hline $\mathrm{N} 2-\mathrm{C} 8-\mathrm{C} 11$ & $120.1(3)$ & $\mathrm{C} 14 \mathrm{~A}-\mathrm{C} 13 \mathrm{~A}-\mathrm{C} 12 \mathrm{~A}$ & $123.5(10)$ \\
\hline $\mathrm{C} 7-\mathrm{C} 8-\mathrm{C} 11$ & $116.1(3)$ & $\mathrm{C} 18 \mathrm{~A}-\mathrm{C} 13 \mathrm{~A}-\mathrm{C} 12 \mathrm{~A}$ & $116.5(10)$ \\
\hline $\mathrm{N} 1-\mathrm{C} 9-\mathrm{C} 10$ & $110.3(3)$ & $\mathrm{C} 15 \mathrm{~A}-\mathrm{C} 14 \mathrm{~A}-\mathrm{C} 13 \mathrm{~A}$ & 120.0 \\
\hline $\mathrm{N} 1-\mathrm{C} 9-\mathrm{H} 9 \mathrm{~A}$ & 109.6 & $\mathrm{C} 15 \mathrm{~A}-\mathrm{C} 14 \mathrm{~A}-\mathrm{C} 11 \mathrm{~A}$ & $117.8(6)$ \\
\hline $\mathrm{C} 10-\mathrm{C} 9-\mathrm{H} 9 \mathrm{~A}$ & 109.6 & $\mathrm{C} 13 \mathrm{~A}-\mathrm{C} 14 \mathrm{~A}-\mathrm{C} 11 \mathrm{~A}$ & $122.2(6)$ \\
\hline $\mathrm{N} 1-\mathrm{C} 9-\mathrm{H} 9 \mathrm{~B}$ & 109.6 & $\mathrm{C} 14 \mathrm{~A}-\mathrm{C} 15 \mathrm{~A}-\mathrm{C} 16 \mathrm{~A}$ & 120.0 \\
\hline $\mathrm{C} 10-\mathrm{C} 9-\mathrm{H} 9 \mathrm{~B}$ & 109.6 & $\mathrm{C} 14 \mathrm{~A}-\mathrm{C} 15 \mathrm{~A}-\mathrm{H} 15 \mathrm{~A}$ & 120.0 \\
\hline $\mathrm{H} 9 \mathrm{~A}-\mathrm{C} 9-\mathrm{H} 9 \mathrm{~B}$ & 108.1 & $\mathrm{C} 16 \mathrm{~A}-\mathrm{C} 15 \mathrm{~A}-\mathrm{H} 15 \mathrm{~A}$ & 120.0 \\
\hline $\mathrm{O} 2-\mathrm{C} 10-\mathrm{N} 3$ & $124.2(3)$ & $\mathrm{C} 15 \mathrm{~A}-\mathrm{C} 16 \mathrm{~A}-\mathrm{C} 17 \mathrm{~A}$ & 120.0 \\
\hline $\mathrm{O} 2-\mathrm{C} 10-\mathrm{C} 9$ & $121.2(3)$ & $\mathrm{C} 15 \mathrm{~A}-\mathrm{C} 16 \mathrm{~A}-\mathrm{H} 16 \mathrm{~A}$ & 120.0 \\
\hline $\mathrm{N} 3-\mathrm{C} 10-\mathrm{C} 9$ & $114.6(3)$ & $\mathrm{C} 17 \mathrm{~A}-\mathrm{C} 16 \mathrm{~A}-\mathrm{H} 16 \mathrm{~A}$ & 120.0 \\
\hline $\mathrm{C} 8-\mathrm{C} 11-\mathrm{C} 12 \mathrm{~A}$ & $113.4(8)$ & $\mathrm{C} 18 \mathrm{~A}-\mathrm{C} 17 \mathrm{~A}-\mathrm{C} 16 \mathrm{~A}$ & 120.0 \\
\hline $\mathrm{C} 8-\mathrm{C} 11-\mathrm{C} 12$ & $112.8(4)$ & $\mathrm{C} 18 \mathrm{~A}-\mathrm{C} 17 \mathrm{~A}-\mathrm{H} 17 \mathrm{~A}$ & 120.0 \\
\hline $\mathrm{C} 8-\mathrm{C} 11-\mathrm{H} 11 \mathrm{~A}$ & 109.0 & $\mathrm{C} 16 \mathrm{~A}-\mathrm{C} 17 \mathrm{~A}-\mathrm{H} 17 \mathrm{~A}$ & 120.0 \\
\hline $\mathrm{C} 12-\mathrm{C} 11-\mathrm{H} 11 \mathrm{~A}$ & 109.0 & $\mathrm{C} 17 \mathrm{~A}-\mathrm{C} 18 \mathrm{~A}-\mathrm{C} 13 \mathrm{~A}$ & 120.0 \\
\hline
\end{tabular}




\begin{tabular}{|c|c|}
\hline $\mathrm{C} 8-\mathrm{C} 11-\mathrm{H} 11 \mathrm{~B}$ & 109.0 \\
\hline $\mathrm{C} 12-\mathrm{C} 11-\mathrm{H} 11 \mathrm{~B}$ & 109.0 \\
\hline $\mathrm{C} 8-\mathrm{N} 2-\mathrm{C} 1-\mathrm{C} 2$ & $178.2(3)$ \\
\hline $\mathrm{C} 8-\mathrm{N} 2-\mathrm{C} 1-\mathrm{C} 6$ & $-0.7(5)$ \\
\hline $\mathrm{N} 2-\mathrm{C} 1-\mathrm{C} 2-\mathrm{C} 3$ & $-179.8(3)$ \\
\hline $\mathrm{C} 6-\mathrm{C} 1-\mathrm{C} 2-\mathrm{C} 3$ & $-0.9(5)$ \\
\hline $\mathrm{C} 1-\mathrm{C} 2-\mathrm{C} 3-\mathrm{C} 4$ & $1.1(6)$ \\
\hline $\mathrm{C} 2-\mathrm{C} 3-\mathrm{C} 4-\mathrm{C} 5$ & $-0.4(6)$ \\
\hline $\mathrm{C} 3-\mathrm{C} 4-\mathrm{C} 5-\mathrm{C} 6$ & $-0.4(6)$ \\
\hline $\mathrm{C} 7-\mathrm{N} 1-\mathrm{C} 6-\mathrm{C} 5$ & $-175.4(3)$ \\
\hline $\mathrm{C} 9-\mathrm{N} 1-\mathrm{C} 6-\mathrm{C} 5$ & $-3.0(5)$ \\
\hline $\mathrm{C} 7-\mathrm{N} 1-\mathrm{C} 6-\mathrm{C} 1$ & $4.5(4)$ \\
\hline $\mathrm{C} 9-\mathrm{N} 1-\mathrm{C} 6-\mathrm{C} 1$ & $176.9(3)$ \\
\hline $\mathrm{C} 4-\mathrm{C} 5-\mathrm{C} 6-\mathrm{N} 1$ & $-179.5(3)$ \\
\hline $\mathrm{C} 4-\mathrm{C} 5-\mathrm{C} 6-\mathrm{C} 1$ & $0.6(5)$ \\
\hline $\mathrm{N} 2-\mathrm{C} 1-\mathrm{C} 6-\mathrm{N} 1$ & $-1.0(5)$ \\
\hline $\mathrm{C} 2-\mathrm{C} 1-\mathrm{C} 6-\mathrm{N} 1$ & $-179.8(3)$ \\
\hline $\mathrm{N} 2-\mathrm{C} 1-\mathrm{C} 6-\mathrm{C} 5$ & $178.9(3)$ \\
\hline $\mathrm{C} 2-\mathrm{C} 1-\mathrm{C} 6-\mathrm{C} 5$ & $0.1(5)$ \\
\hline $\mathrm{C} 6-\mathrm{N} 1-\mathrm{C} 7-\mathrm{O} 1$ & $174.3(3)$ \\
\hline $\mathrm{C} 9-\mathrm{N} 1-\mathrm{C} 7-\mathrm{O} 1$ & $1.6(5)$ \\
\hline $\mathrm{C} 6-\mathrm{N} 1-\mathrm{C} 7-\mathrm{C} 8$ & $-5.9(4)$ \\
\hline $\mathrm{C} 9-\mathrm{N} 1-\mathrm{C} 7-\mathrm{C} 8$ & $-178.6(3)$ \\
\hline $\mathrm{C} 1-\mathrm{N} 2-\mathrm{C} 8-\mathrm{C} 7$ & $-1.0(5)$ \\
\hline $\mathrm{C} 1-\mathrm{N} 2-\mathrm{C} 8-\mathrm{C} 11$ & $-178.6(3)$ \\
\hline $\mathrm{O} 1-\mathrm{C} 7-\mathrm{C} 8-\mathrm{N} 2$ & $-176.0(3)$ \\
\hline $\mathrm{N} 1-\mathrm{C} 7-\mathrm{C} 8-\mathrm{N} 2$ & $4.2(5)$ \\
\hline $\mathrm{O} 1-\mathrm{C} 7-\mathrm{C} 8-\mathrm{C} 11$ & $1.8(4)$ \\
\hline $\mathrm{N} 1-\mathrm{C} 7-\mathrm{C} 8-\mathrm{C} 11$ & $-178.1(3)$ \\
\hline $\mathrm{C} 7-\mathrm{N} 1-\mathrm{C} 9-\mathrm{C} 10$ & $88.2(3)$ \\
\hline $\mathrm{C} 6-\mathrm{N} 1-\mathrm{C} 9-\mathrm{C} 10$ & $-84.6(3)$ \\
\hline $\mathrm{N} 4-\mathrm{N} 3-\mathrm{C} 10-\mathrm{O} 2$ & $-2.4(5)$ \\
\hline $\mathrm{N} 4-\mathrm{N} 3-\mathrm{C} 10-\mathrm{C} 9$ & $175.5(3)$ \\
\hline $\mathrm{N} 1-\mathrm{C} 9-\mathrm{C} 10-\mathrm{O} 2$ & $-3.5(4)$ \\
\hline $\mathrm{N} 1-\mathrm{C} 9-\mathrm{C} 10-\mathrm{N} 3$ & $178.6(3)$ \\
\hline
\end{tabular}

C17A-C18A-H18A

C13A-C18A-H18A

$\mathrm{N} 2-\mathrm{C} 8-\mathrm{C} 11-\mathrm{C} 12 \mathrm{~A}$

$\mathrm{C} 7-\mathrm{C} 8-\mathrm{C} 11-\mathrm{C} 12 \mathrm{~A}$

$\mathrm{N} 2-\mathrm{C} 8-\mathrm{C} 11-\mathrm{C} 12$

$\mathrm{C} 7-\mathrm{C} 8-\mathrm{C} 11-\mathrm{C} 12$

$\mathrm{C} 8-\mathrm{C} 11-\mathrm{C} 12-\mathrm{C} 13$

$\mathrm{C} 11-\mathrm{C} 12-\mathrm{C} 13-\mathrm{C} 14$

$\mathrm{C} 11-\mathrm{C} 12-\mathrm{C} 13-\mathrm{C} 18$

$\mathrm{C} 18-\mathrm{C} 13-\mathrm{C} 14-\mathrm{C} 15$

$\mathrm{C} 12-\mathrm{C} 13-\mathrm{C} 14-\mathrm{C} 15$

$\mathrm{C} 18-\mathrm{C} 13-\mathrm{C} 14-\mathrm{Cl1}$

$\mathrm{C} 12-\mathrm{C} 13-\mathrm{C} 14-\mathrm{Cl1}$

$\mathrm{C} 13-\mathrm{C} 14-\mathrm{C} 15-\mathrm{C} 16$

$\mathrm{C} 11-\mathrm{C} 14-\mathrm{C} 15-\mathrm{C} 16$

$\mathrm{C} 14-\mathrm{C} 15-\mathrm{C} 16-\mathrm{C} 17$

$\mathrm{C} 15-\mathrm{C} 16-\mathrm{C} 17-\mathrm{C} 18$

$\mathrm{C} 16-\mathrm{C} 17-\mathrm{C} 18-\mathrm{C} 13$

$\mathrm{C} 14-\mathrm{C} 13-\mathrm{C} 18-\mathrm{C} 17$

$\mathrm{C} 12-\mathrm{C} 13-\mathrm{C} 18-\mathrm{C} 17$

$\mathrm{C} 8-\mathrm{C} 11-\mathrm{C} 12 \mathrm{~A}-\mathrm{C} 13 \mathrm{~A}$

$\mathrm{C} 11-\mathrm{C} 12 \mathrm{~A}-\mathrm{C} 13 \mathrm{~A}-\mathrm{C} 14 \mathrm{~A}$

$\mathrm{C} 11-\mathrm{C} 12 \mathrm{~A}-\mathrm{C} 13 \mathrm{~A}-\mathrm{C} 18 \mathrm{~A}$

$\mathrm{C} 18 \mathrm{~A}-\mathrm{C} 13 \mathrm{~A}-\mathrm{C} 14 \mathrm{~A}-\mathrm{C} 15 \mathrm{~A}$

$\mathrm{C} 12 \mathrm{~A}-\mathrm{C} 13 \mathrm{~A}-\mathrm{C} 14 \mathrm{~A}-\mathrm{C} 15 \mathrm{~A}$

$\mathrm{C} 18 \mathrm{~A}-\mathrm{C} 13 \mathrm{~A}-\mathrm{C} 14 \mathrm{~A}-\mathrm{C} 11 \mathrm{~A}$

$\mathrm{C} 12 \mathrm{~A}-\mathrm{C} 13 \mathrm{~A}-\mathrm{C} 14 \mathrm{~A}-\mathrm{C} 11 \mathrm{~A}$

$\mathrm{C} 13 \mathrm{~A}-\mathrm{C} 14 \mathrm{~A}-\mathrm{C} 15 \mathrm{~A}-\mathrm{C} 16 \mathrm{~A}$

$\mathrm{C} 11 \mathrm{~A}-\mathrm{C} 14 \mathrm{~A}-\mathrm{C} 15 \mathrm{~A}-\mathrm{C} 16 \mathrm{~A}$

$\mathrm{C} 14 \mathrm{~A}-\mathrm{C} 15 \mathrm{~A}-\mathrm{C} 16 \mathrm{~A}-\mathrm{C} 17 \mathrm{~A}$

$\mathrm{C} 15 \mathrm{~A}-\mathrm{C} 16 \mathrm{~A}-\mathrm{C} 17 \mathrm{~A}-\mathrm{C} 18 \mathrm{~A}$

$\mathrm{C} 16 \mathrm{~A}-\mathrm{C} 17 \mathrm{~A}-\mathrm{C} 18 \mathrm{~A}-\mathrm{C} 13 \mathrm{~A}$

$\mathrm{C} 14 \mathrm{~A}-\mathrm{C} 13 \mathrm{~A}-\mathrm{C} 18 \mathrm{~A}-\mathrm{C} 17 \mathrm{~A}$

$\mathrm{C} 12 \mathrm{~A}-\mathrm{C} 13 \mathrm{~A}-\mathrm{C} 18 \mathrm{~A}-\mathrm{C} 17 \mathrm{~A}$
120.0

120.0

$-97.9(9)$

84.3 (9)

-115.1 (4)

67.1 (4)

179.0 (4)

-86.4 (5)

89.8 (4)

0.0

176.2 (5)

178.8 (4)

$-5.0(5)$

0.0

-178.9 (4)

0.0

0.0

0.0

0.0

-176.4 (4)

176.3 (8)

77.5 (13)

-101.4 (12)

0.0

-178.9 (9)

-178.8 (9)

2.3 (11)

0.0

178.9 (8)

0.0

0.0

0.0

0.0

179.0 (8)

Hydrogen-bond geometry $\left(\AA,{ }^{\circ}\right)$

\begin{tabular}{lllll}
\hline$D-\mathrm{H} \cdots A$ & $D-\mathrm{H}$ & $\mathrm{H} \cdots A$ & $D \cdots A$ & $D-\mathrm{H} \cdots A$ \\
\hline $\mathrm{N} 3-\mathrm{H} 3 A \cdots \mathrm{O} 2^{\mathrm{i}}$ & 0.91 & 1.96 & $2.842(4)$ & 163 \\
$\mathrm{~N} 4-\mathrm{H} 4 A \cdots \mathrm{N} 2^{\mathrm{ii}}$ & 0.91 & 2.35 & $3.256(4)$ & 175 \\
$\mathrm{~N} 4-\mathrm{H} 4 B \cdots \mathrm{C}^{\mathrm{ii}}$ & 0.91 & 2.92 & $3.539(3)$ & 126 \\
\hline
\end{tabular}

Symmetry codes: (i) $x, y+1, z$; (ii) $-x+1,-y+1, z-1 / 2$. 